\title{
Criminologie
}

\section{L’absence temporaire dans les établissements de détention du Québec}

\section{Pierre Landreville}

Volume 28, numéro 1, 1995

L'histoire de l'enfermement et des populations pénales

URI : https://id.erudit.org/iderudit/017368ar

DOI : https://doi.org/10.7202/017368ar

Aller au sommaire du numéro

Éditeur(s)

Les Presses de l'Université de Montréal

ISSN

0316-0041 (imprimé)

1492-1367 (numérique)

Découvrir la revue

Citer cet article

Landreville, P. (1995). L'absence temporaire dans les établissements de détention du Québec. Criminologie, 28(1), 139-147.

https://doi.org/10.7202/017368ar
Résumé de l'article

In this article the author exposes the situation of prison overcrowding in facilities in the province of Québec. He brings forth the fact that leave of absences have been used to respond to this situation and states that these programs are acceptable means of managing carcéral populations. He bases his statements on the spacial comparison of the rates of leave of absences and the rates of overcrowding between 1989 and 1992, and this by various administrative regions of Québec. 


\section{L'ABSENCE TEMPORAIRE DANS LES ÉTABLISSEMENTS DE DÉTENTION DU QUÉBEC Pierre Landreville}

In this article the author exposes the situation of prison overcrowding in facilities in the province of Québec. He brings forth the fact that leave of absences have been used to respond to this situation and states that these programs are acceptable means of managing carceral populations. He bases his statements on the spacial comparison of the rates of leave of absences and the rates of overcrowding between 1989 and 1992, and this by various administrative regions of Québec.

La population des établissements carcéraux a augmenté considérablement depuis vingt ans, tant au Canada et aux États-Unis que dans la plupart des autres pays occidentaux. Au Canada, il y avait 19668 personnes incarcérées en 1972, soit un taux de détention de 90 pour 100000 habitants (Waller et Chan, 1974), alors qu'il y en avait plus de 30000 en $1991-1992$, soit 114 pour 100000 habitants. Aux États-Unis, la population carcérale est passée de 406531 en 1970 (taux de 200) (Waller et Chan, 1974), à un peu plus 1200000 en 1991 (taux de plus de 500) (Christie, 1993, p. 82). L'augmentation de ces populations durant les années 1980 a été si rapide, surtout aux États-Unis, qu'elle a causé un très sérieux problème de surpopulation.

Cette surpopulation augmente la tension dans les prisons, crée parfois des problèmes de sécurité pour les gardiens et les détenus et diminue la quantité et la qualité des programmes correctionnels. Dans plusieurs cas, elle provoque des conditions de détention qui mettent en péril le respect des droits fondamentaux et vont à l'encontre de la dignité humaine. Aux ÉtatsUnis, la situation est telle dans certains systèmes correctionnels que les tribunaux sont intervenus pour déclarer que les conditions de détention étaient inconstitutionnelles. Des auteurs ont pu écrire que l'incarcération était devenue «a deviant form of social control» (Pontell et Welsh, 1994. p. 18). .

Même si la situation est beaucoup moins grave au Canada que chez nos voisins du sud, elle est néanmoins devenue préoccupante et a stimulé des initiatives de la part des administrateurs pénitentiaires telles la double occupation cellulaire et l'accroissement des absences temporaires. C'est de cette

1. Co-directeur du Groupe de recherche et d'analyse sur les politiques et les pratiques pénales, École de criminologie, Centre international de criminologie comparée, Université de Montréal, C.P. 6128, Succ. Centre-Ville, Montréal QC H3C $3 \mathrm{~J} 7$. 
dernière mesure et de son utilisation dans le cas de courtes peines d'emprisonnement ( 2 ans et moins) qu'il sera question dans cet article.

Nous y aborderons quatre points : 1) en premier lieu, il sera brièvement question des diverses façons de faire face à la surpopulation carcérale; 2) nous traiterons de la surpopulation dans les établissements de détention de la province de Québec; 3 ) nous démontrerons que les absences temporaires ont été utilisées pour faire face à cette situation de surpopulation; 4) enfin, nous soutiendrons que ces programmes sont un moyen acceptable de gérer les populations carcérales.

\section{LES RÉPONSES À LA SURPOPULATION}

La population carcérale à un moment donné (stock ${ }^{2}$ ) est déterminée par le nombre des admissions (flux) et la durée des séjours ( $\mathrm{S}=\mathrm{F} \times \mathrm{D}$ ).

Les stratégies pour réduire la surpopulation carcérale sont donc de trois ordres : a) celles portant sur les admissions; b) celles portant sur la durée des peines ou des séjours ; c) l'augmentation de la capacité carcérale.

a) Les stratégies pour réduire les admissions sont surtout d'ordre législatif. Elles consistent en l'abolition de peines minimales ou obligatoires d'emprisonnement, la mise sur pied de solutions de rechange à l'incarcération (Landreville, 1994; McMahon, 1993), l'assouplissement des lois favorisant la remise en liberté pendant le procès. Il peut aussi s'agir de mesures administratives telles les listes d'attente pour l'admission en prison que l'on retrouve en Norvège (Christie, 1993, p. 35) ou aux Pays-Bas (Downes, 1982).

b) La durée des peines ou des séjours en prison, qui est probablement le facteur le plus important pour la population carcérale (Young et Brown, 1993, p. 44; Khun, 1994), peut être contrôlée ou écourtée à l'aide de stratégies qui agissent lors du prononcé des peines, comme les lignes directrices sur la détermination de la peine (sentencing guidelines) du Minnesota et de Washington (Tonry, 1991, p. 311-13).

Mais la plupart des mesures touchant la durée des séjours favorisent des libérations anticipées et constituent ce que l'on a appelé des back-end methods. Dans certains cas, il s'agira de mesures exceptionnelles telles l'amnistie (Tournier, 1992) ou des modifications législatives concernant la durée de la rémission des peines. Les pratiques de libération conditionnelle ont aussi un impact important sur la durée des séjours et jouent un rôle non négligeable, mais pas toujours reconnu, dans la régulation des populations carcérales. Enfin, des mesures prises par les administrateurs, telles les permissions de s'absenter, ordinairement vers la fin de la sentence, pour participer à des programmes de travail, d'éducation ou de réinsertion

2. Selon l'expression consacrée du Conseil de l'Europe. 
sociale, jouent aussi sur la durée des séjours et contribuent à réduire ou à contrôler la surpopulation carcérale.

c) L'augmentation de la capacité carcérale peut être réalisée par la construction de nouvelles prisons, comme cela se passe dans la plupart des États américains (Vaugh, 1993) et dans de nombreux pays européens. On peut aussi instituer la double ou la triple occupation cellulaire, comme on l'a fait récemment dans les pénitenciers canadiens.

\section{LA SITUATION DANS LES ÉTABLISSEMENTS DE DÉTENTION PROVINCIAUX DU QUÉBEC}

En 1991-1992, il y avait en moyenne 30727 personnes incarcérées au Canada (taux de détention de 114 pour 100000 habitants) dont 18944 dans des établissements provinciaux et 11783 dans des pénitenciers fédéraux. Au Canada, il y avait 4947 prévenus (16\% de toutes les personnes incarcérées), et par ailleurs, $33 \%$ des personnes condamnées admises dans des établissements provinciaux l'avaient été pour non-paiement d'amende (Statistique Canada, 1993).

Au Québec, dans les établissements de détention provinciaux en 1991 1992, il y avait en moyenne 4584 personnes inscrites et 3349 personnes présentes. Il y avait donc en moyenne 1235 personnes absentes, soit environ $25 \%$ de la population inscrite. Compte tenu de la capacité carcérale normale (soit 3156 places), le taux d'occupation moyen (population présente/capacité normale) était de 1,06 et le taux de surpopulation (population inscrite/capacité normale) de 1,45.

Même si la loi permet aux administrateurs d'autoriser des absences temporaires pour des raisons humanitaires ou de réinsertion sociale depuis 1969, ce n'est qu'en 1981, lorsque la population moyenne inscrite (2993) a dépassé la capacité normale ( 2922 ), que la moyenne quotidienne d'absences temporaires a dépassé $100(101,5)$. Celle-ci est passée à 671 en 1986, alors que la population moyenne inscrite était de 3267 par rapport à une capacité normale de 2737 , puis à 1235 en 1991-1992, comme nous venons de le voir.

\section{3- LA SURPOPULATION ET LES ABSENCES TEMPORAIRES AU QUÉBEC DE 1989 À 1992}

Jusqu'en 1992, la loi permettait aux administrateurs d'accorder aux personnes condamnées des absences temporaires pour des raisons humanitaires ou de réinsertion sociale, pour une période maximale de 15 jours, lorsqu'elles avaient purgé le tiers de leur peine. En réalité, en raison de la surpopulation, ils en étaient venus à accorder ces mesures à des personnes qui n'avaient pas complété le tiers de leur peine et à interpréter la loi de façon très large en accordant plusieurs absences de manière consécutive, de 
sorte que, dans certains cas, la période d'absence pouvait se prolonger durant plusieurs mois.

L'utilisation des absences temporaires pour faire face à la surpopulation était "généralement connue " mais n'a jamais été démontrée empiriquement. C'est ce que nous allons tenter de faire, en utilisant les quelques données disponibles à ce sujet.

Pour établir que les absences temporaires n'ont pas été accordées dans le seul but de favoriser la réinsertion sociale mais plutôt comme moyen de gérer la surpopulation, on aurait pu construire des séries chronologiques pour démontrer que sur plusieurs années, les absences temporaires varient en fonction des taux de surpopulation. Malheureusement, ces données n'existent pas pour une longue période. Afin de pallier cette lacune, nous avons plutôt établi les variations spatiales de ces deux variables, selon 11 régions socio-administratives de la province de Québec.

Si les absences temporaires sont accordées uniquement en vue de la réinsertion sociale, elles devraient se répartir également dans les diverses régions. Par contre, si on les accorde surtout pour gérer la surpopulation, les taux d'absences temporaires ( $n$ jours d'absences/ $n$ jours de la population inscrite par année) devraient être fortement corrélés aux taux de surpopulation (population inscrite/capacité normale) par région.

Tableau 1

Taux de surpopulation et d'absences temporaires dans les établissements de détention du Québec, selon les régions, 1991-1992

\begin{tabular}{lcccc|rrrr}
\hline Région & $\begin{array}{c}\text { Population } \\
\text { inscrite }\end{array}$ & $\begin{array}{c}\text { Capacité } \\
\text { normale }\end{array}$ & $\begin{array}{c}\text { Taux de } \\
\text { surpopu- } \\
\text { lation }\end{array}$ & Rang & $\begin{array}{c}\text { Abs. temp. } \\
\text { (Jours) }\end{array}$ & $\begin{array}{r}\text { Pop. insc. } \\
\text { (Jours) }\end{array}$ & $\begin{array}{r}\text { Taux } \\
\text { abs. } \\
\text { temp. }\end{array}$ & Rang \\
\hline 01 & 130 & 91 & 1,43 & 9 & 12535 & 47580 & 0,26 & 7,5 \\
02 & 143 & $82^{*}$ & 1,74 & 3 & 13142 & 52338 & 0,25 & 9 \\
03 & 706 & 475 & 1,49 & 7 & 70657 & 258396 & 0,27 & 6 \\
04 & 225 & 107 & 2,10 & 1 & 35504 & 82350 & 0,43 & 1 \\
05 & 149 & 119 & 1,25 & 10 & 11070 & 54534 & 0,20 & 10 \\
$06 \mathrm{a}$ & 1245 & 827 & 1,50 & 6 & 138241 & 455670 & 0,30 & 5 \\
$06 \mathrm{~b}$ & 253 & 147 & 1,72 & 4 & 32146 & 92598 & 0,35 & 3 \\
$06 \mathrm{c}$ & 276 & 174 & 1,59 & 5 & 25980 & 101016 & 0,26 & 7,5 \\
07 & 129 & $73 * *$ & 1,77 & 2 & 18789 & 47214 & 0,40 & 2 \\
08 & 118 & $82 * *$ & 1,44 & 8 & 14042 & 43188 & 0,32 & 4 \\
09 & 91 & 84 & 1,08 & 11 & 3471 & 33306 & 0,10 & 11 \\
\hline
\end{tabular}

* Plus de 40 places « occasionnelles ».

** Aucune place « occasionnelle».

$\mathrm{RHO}=0,71$; 
Au tableau 1, dans la région 6a, nous avons exclu le Centre de prévention de Montréal, puisqu'on ne peut accorder d'absences temporaires pour des raisons de réinsertion sociale à des personnes prévenues, et dans la région $6 \mathrm{c}$ nous n'avons pas tenu compte du centre de Waterloo, centre à sécurité minimale, où la gestion de la population peut être particulière. Dans ce tableau, on constate une forte relation entre les taux de surpopulation et les taux d'absences. Il y a moins de $5 \%$ de chances que la corrélation de rang soit due au hasard. À deux exceptions près, les régions 02 et 08 , il y a une relation presque parfaite entre ces deux taux selon les régions.

Les données des deux régions atypiques renforcent même notre hypothèse. Ces deux régions ont la même capacité carcérale «normale », soit 82 , mais dans l'une (02) d'elles, il y a 40 places « occasionnelles ${ }^{3}$ » qui peuvent s'ajouter à la capacité normale, tandis que dans l'autre (08), il n'y a aucune place «occasionnelle». On peut alors très bien comprendre que dans la première $(02)$, un fort taux de surpopulation $(1,74)$ exerce une pression modérée sur la capacité carcérale totale et qu'en conséquence on retrouve un taux d'absences temporaires plutôt faible $(0,25)$. Par contre, un taux plus faible de surpopulation $(1,44)$ dans la région 08 devient plus intolérable, puisqu'il n'y a aucune marge de manæuvre, ce qui engendre un taux d'absences relativement fort $(0,32)$. N'eût été cette anomalie dans la détermination des capacités carcérales, la corrélation de rang aurait été presque parfaite.

Les données des années 1989-1990 et 1990-1991 (tableaux 2 et 3) corroborent celles présentées pour 1991-1992. Il n'y a donc aucun doute que durant ces trois années, les administrateurs des établissements de détention du Québec ont utilisé les absences temporaires pour diminuer la pression exercée par l'excédent de population sur leur établissement.

Tableau 2

Taux de surpopulation et d'absences temporaires dans les établissements de détention du Québec, selon les régions, 1990-1991

\begin{tabular}{lcccc|rrrr}
\hline Région & $\begin{array}{c}\text { Population } \\
\text { inscrite }\end{array}$ & $\begin{array}{c}\text { Capacité } \\
\text { normale }\end{array}$ & $\begin{array}{c}\text { Taux de } \\
\text { surpopu- } \\
\text { lation }\end{array}$ & Rang & $\begin{array}{c}\text { Abs. temp. } \\
\text { (Jours) }\end{array}$ & $\begin{array}{c}\text { Pop. insc. } \\
\text { (Jours) }\end{array}$ & $\begin{array}{r}\text { Taux } \\
\text { abs. } \\
\text { temp. }\end{array}$ & Rang \\
\hline 01 & 100 & 91 & 1,10 & 10 & 5718 & 36600 & 0,16 & 9,5 \\
02 & 117 & $84^{*}$ & 1,39 & 4 & 11691 & 42822 & 0,27 & 4 \\
03 & 637 & 511 & 1,25 & 8 & 50679 & 233142 & 0,22 & 8 \\
04 & 218 & 107 & 2,04 & 1 & 31188 & 79788 & 0,39 & 1 \\
05 & 104 & 76 & 1,37 & 5 & 9617 & 38064 & 0,25 & 5 \\
$06 \mathrm{a}$ & 1135 & 827 & 1,37 & 5 & 93785 & 415410 & 0,23 & 7 \\
$06 \mathrm{~b}$ & 213 & 147 & 1,45 & 3 & 19513 & 77958 & 0,25 & 5 \\
\hline
\end{tabular}

3. «Lits supplémentaires que l'établissement est capable de foumir occasionnellement pour la garde de la clientèle.» 


\begin{tabular}{lrccc|cccc}
\hline Région & $\begin{array}{c}\text { Population Capacité } \\
\text { inscrite }\end{array}$ & $\begin{array}{c}\text { Taux de } \\
\text { normale } \\
\text { strpopu- } \\
\text { lation }\end{array}$ & Rang & $\begin{array}{c}\text { Abs. temp. } \\
\text { (Jours) }\end{array}$ & $\begin{array}{c}\text { Pop. insc. } \\
\text { (Jours) }\end{array}$ & $\begin{array}{c}\text { Taux } \\
\text { abs. } \\
\text { temp. }\end{array}$ & Rang \\
\hline $06 \mathrm{c}$ & 231 & 174 & 1,33 & 7 & 13285 & 84546 & 0,16 & 9,5 \\
07 & 110 & $73^{* *}$ & 1,51 & 2 & 13095 & 40260 & 0,33 & 3 \\
08 & 95 & $82^{* *}$ & 1,16 & 9 & 12900 & 34770 & 0,37 & 2 \\
09 & 82 & 84 & 0,98 & 11 & 3642 & 30012 & 0,12 & 11 \\
\hline
\end{tabular}

* Plus de 40 places « occasionnelles ».

** Aucune place « occasionnelle».

RHO $=0,71$;

Tableau 3

Taux de surpopulation et d'absences temporaires dans les établissements de détention du Québec, selon les régions, 1989-1990

\begin{tabular}{lcccc|rrrr}
\hline Région & $\begin{array}{c}\text { Population } \\
\text { inscrite }\end{array}$ & $\begin{array}{c}\text { Capacité } \\
\text { normale }\end{array}$ & $\begin{array}{c}\text { Taux de } \\
\text { surpopu- } \\
\text { Jation }\end{array}$ & Rang & $\begin{array}{c}\text { Abs. temp. } \\
\text { (Jours) }\end{array}$ & $\begin{array}{c}\text { Pop. insc. } \\
\text { (Jours) }\end{array}$ & $\begin{array}{c}\text { Taux } \\
\text { abs. } \\
\text { temp. }\end{array}$ & Rang \\
\hline 01 & 99 & 91 & 1,09 & 11 & 6292 & 36234 & 0,17 & 9,5 \\
02 & 128 & $84^{*}$ & 1,52 & 5 & 14576 & 46848 & 0,31 & 5 \\
03 & 664 & 511 & 1,30 & 8 & 55479 & 243024 & 0,23 & 7 \\
04 & 186 & 107 & 1,74 & 1 & 27947 & 68076 & 0,41 & 1 \\
05 & 100 & 76 & 1,32 & 7 & 14061 & 36600 & 0,38 & 2 \\
$06 \mathrm{a}$ & 1322 & 827 & 1,60 & 3 & 146817 & 483852 & 0,30 & 6 \\
$06 \mathrm{~b}$ & 197 & 147 & 1,34 & 6 & 15242 & 72102 & 0,21 & 8 \\
$06 \mathrm{c}$ & 217 & 174 & 1,25 & 9 & 13577 & 79422 & 0,17 & 9,5 \\
07 & 118 & $73^{* *}$ & 1,62 & 2 & 14457 & 43188 & 0,33 & 3,5 \\
08 & 91 & $58 * *$ & 1,57 & 4 & 10969 & 33306 & 0,33 & 3,5 \\
09 & 92 & 84 & 1,10 & 10 & 3766 & 33672 & 0,11 & 11 \\
\hline
\end{tabular}

* Plus de 40 places « occasionnelles".

** Aucune place «occasionnelle».

$\mathrm{RHO}=0,79$;

\section{L'ABSENCE TEMPORAIRE COMME MODE DE GESTION DE LA POPULATION CARCÉRALE}

L'utilisation des mesures de libération anticipée, même sous surveillance, pour répondre à la surpopulation carcérale a été critiquée de plusieurs points de vue.

Selon Austin (1986, p. 405), par exemple, il s'agit d'une "extremely controversial approach to curbing prison crowding ", parce que, d'une part, la libération anticipée diminue l'effet de neutralisation ce qui peut mettre le public en danger. D'autre part, ajoute-t-il, «early release program might adversely affect general deterrence» (ibid., p. 405) et, en 
troisième lieu, la population peut perdre confiance dans le système pénal qu'elle percevra comme moins efficace. $D^{\prime}$ autres pourraient ajouter que cette modification de la durée des peines par les administrateurs va à l'encontre de la rétribution, puisqu'elle transforme la «peine méritée » (just desert).

En plus de ces critiques qui portent sur les finalités de la peine d'emprisonnement, on peut aussi avancer qu'il s'agit d'un moyen peu adéquat pour régulariser la population carcérale. Austin formule cet argument en ces termes: «Early release can provide no more than a short-term remedy for prison crowding. [...] Once policymakers enact legislation and policies that are affordable, the need for early release will disappear. » (Austin, 1986, p. 410.)

Ces critiques ne nous semblent pas fondées en ce qui concerne la discussion de principes et l'inadéquation de ces mesures du point de vue pratique, particulièrement dans le cas de prisons pour de courtes peines où il y a une très grande fluctuation des populations. Il semble, au contraire, qu'il s'agit de pratiques légitimes et nécessaires qui respectent des principes de politique pénale importants: les principes de modération, de justice et d'humanité.

Quant aux finalités de la peine, il va tout d'abord de soi qu'une libération anticipée diminue la neutralisation. Mais lorsqu'il est question de l'absence temporaire, la mesure s'applique sur une partie relativement courte de la peine, à la suite d'un processus de sélection, même sommaire, et la personne libérée demeure sous la surveillance d'agents correctionnels. On peut croire que les personnes libérées de cette façon sont de meilleurs « risques » et que les infractions, généralement mineures, qu'elles commettront un peu plus tôt qu'elles ne l'auraient fait constituent une infime partie de la criminalité et ne constituent pas un danger indô pour la population.

On ne peut pas soutenir sérieusement qu'une diminution de quelques semaines ou quelques mois de l'incarcération chez une partie relativement faible de la population carcérale aura un impact sur la dissuasion générale. Il est aussi difficile d'affirmer que de telles modifications de la durée des peines portent sérieusement atteinte au principe de la peine méritée. Il faudrait alors postuler que les peines imposées par les juges, généralement suite à une proposition négociée entre la défense et la poursuite, constituent infailliblement le « juste dû ».

L'argument selon lequel des mesures de libération anticipée pour contrôler la population carcérale seraient inutiles si les décideurs mettaient en place des législations et des politiques «responsables» qui tiennent compte des moyens de réalisation de ces décisions, en particulier la capacité carcérale, semble tout à fait farfelu. Il est en effet généralement admis 
que le système pénal n'est pas vraiment un système, que ses diverses parties et les différents acteurs qui y œuvrent sont relativement indépendants, qu'ils poursuivent des objectifs internes souvent très différents des intentions et des buts des "législateurs » et des planificateurs, et qu'en réalité il s'agit d'un processus relativement incontrôlé et incontrôlable. Des mesures de régulation d'un "sous-système » comme les prisons, et même d'un élément de celui-ci, comme une ou quelques prisons en particulier, seront toujours indispensables. Ces mesures de régulation sont d'autant plus nécessaires lorsqu'il est question de prisons pour courtes peines et d'incarcération des personnes prévenues où il y a généralement des fluctuations de populations mensuelles, hebdomadaires et même journalières très importantes, tant dans l'ensemble du système que dans chaque région et chaque établissement.

Au Québec, par exemple, il y avait en 1991-1992 une population moyenne inscrite de 4584 personnes dans les établissements de détention sous juridiction provinciale, alors qu'à un jour donné, le 6 mai 1992, la population inscrite était de 6009 personnes, soit $1425(31 \%)$ de plus que la population annuelle moyenne. Par ailleurs, si le taux de surpopulation (population inscrite/capacité normale) était de 1,45, il variait de 1,08 à 2,10 selon les régions (tableau 1). Ces variations seraient encore plus prononcées si on disposait de données journalières ou par établissement.

On comprend aisément qu'aucun système ne peut être construit, à des coûts acceptables, pour répondre à des situations exceptionnelles, qui se situent à $40 \%$ ou $50 \%$ au-dessus de son taux de fonctionnement moyen. Dans le cas des prisons pour courtes peines, qui font face à des situations semblables, il doit nécessairement y avoir des processus de régulation efficaces, telles les mesures de libération anticipée, en particulier les absences temporaires accordées par les autorités administratives des prisons.

Ces mécanismes sont aussi parfois indispensables pour maintenir des conditions de détention acceptables. En effet, presque partout dans le monde, la plupart des personnes incarcérées le sont dans des prisons vétustes, sans travail valorisant, et elles ne participent généralement pas à des programmes correctionnels. L'augmentation, souvent phénoménale, des populations carcérales à laquelle on a assisté dans de nombreux pays durant la dernière décennie, a parfois conduit à la détérioration des conditions de détention, au point qu'elles sont tout à fait inacceptables et peuvent même être considérées comme cruelles dans certaines prisons, comme en font foi, par exemple, des décisions judiciaires américaines.

Partout, les administrateurs doivent prioritairement mettre en place des politiques et des programmes pour mieux faire respecter, au sein du monde carcéral, les valeurs fondamentales de nos sociétés qui sont aussi à la base 
des systèmes de l'administration de la justice pénale, comme la justice, l'équité, le respect de la dignité des personnes.

Il faut reconnaître que des mesures de remise en liberté autorisées par les autorités carcérales, tout comme la remise en liberté conditionnelle, peuvent jouer ce rôle régulateur. Ces mesures, qui reposent généralement sur un processus de sélection et qui veillent à favoriser la réinsertion sociale, sont des mesures de régulation beaucoup plus adéquates que des mesures d'amnistie ou de remise de peine ponctuelle à toutes les personnes incarcérées, auxquelles on a parfois recours pour pallier des situations catastrophiques. Ce mode de contrôle du volume de la population carcérale est aussi préférable au refoulement en amont, dans des systèmes carcéraux encore moins adéquats (comme les jails aux États-Unis), de ceux que l'on ne peut admettre.

\section{BIBLIOGRAPHIE}

AUSTIN, J. (1986), "Using Early Release to Relieve Prison Crowding; A Dilemma in Public Policy ", Crime \& Delinquency, vol. 32, $n^{\circ} 4$, pp. 404-502.

CHRISTIE, N. (1993), Crime Control as Industry, Londres, Routledge.

DOWNES, D. (1983), «The Origins and Consequences of Dutch Penal Policy Since 1945 », The British Journal of Criminology, vol. 22, $n^{\circ} 4$, pp. 325-362.

KUHN, A. (1994), "Le nombre carcéral», Revue de criminologie et de police technique, vol. XLVII, $n^{\circ} 3$, pp. 310-321.

LANDREVILLE, P. (1994), « Compensatory Work Program, a Way of Limiting Prison Use ? The Québec's Experience », The Howard Journal, (à paraittre).

McMAHON, M. (1993), The Persistent Prison? Rethinking Decarceration and Penal Reform, Toronto, Toronto University Press.

PONTELL, H. et W. WELSH (1994), "Incarceration as a Deviant Form of Social Control », Crime \& Delinquency, vol. 40, $\mathrm{n}^{\circ}$ 1, pp. 18-36.

TONRY, M. (1991), "The Politics and Processes of Sentencing Commissions», Crime \& Delinquency, vol. 37, pp. 307-329.

TOURNIER, P. (1992), Démographie des prisons françaises. Toujours plus?, Études et données pénales, $\mathrm{n}^{\circ} 64$, Paris, C.E.S.D.I.P.

STATISTIQUE CANADA, (1993), Services correctionnels pour adultes au Canada, 1991-1992, Ottawa, Centre canadien de la statistique juridique.

VAUGHN, M. S. (1993), «Listening to the Experts : A National Study of Correctional Administrators' Responses to Prison Overcrowding ", Criminal Justice Review, vol. $18, \mathrm{n}^{\circ} 1, \mathrm{pp} .12-25$.

YOUNG, W. et M. BROWN (1993), "Cross-national Comparisons of Imprisonment », Crime and Justice. A Review of Research, vol. 17, pp. 1-49.

WALLER, I. et J. CHAN (1974), « Prison Use : A Canadian and International Comparison », Criminal Law Quarterly, vol. 17, $\mathrm{n}^{\circ} 1, \mathrm{pp} .47-71$. 